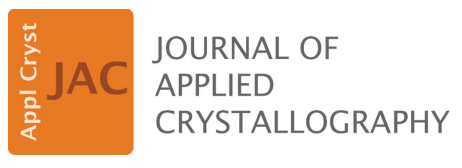

ISSN $1600-5767$

Received 30 March 2016

Accepted 15 October 2016

Edited by M. Gradzielski, Technische

Universität Berlin, Germany

${ }^{1}$ This article will form part of a virtual special issue of the journal, presenting some highlights of the 16th International Conference on SmallAngle Scattering (SAS2015).

Keywords: small-angle neutron scattering (SANS); contrast variation; dynamic nuclear polarization; ternary mixtures; nanocomposites.

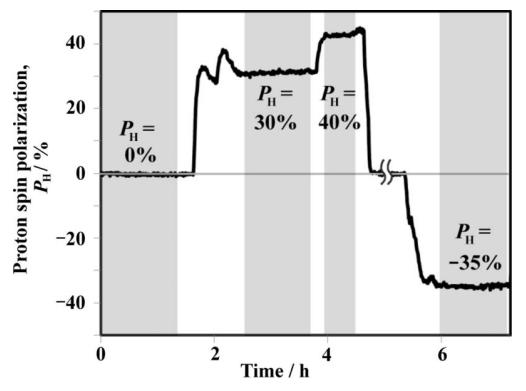

OPEN $\odot$ ACCESS

\section{Contrast variation by dynamic nuclear polarization and time-of-flight small-angle neutron scattering. I. Application to industrial multi-component nanocomposites ${ }^{1}$}

\author{
Yohei Noda, ${ }^{\text {a* }}$ Satoshi Koizumi, ${ }^{\text {a* }}$ Tomomi Masui, ${ }^{\text {b }}$ Ryo Mashita, ${ }^{\text {b }}$ Hiroyuki \\ Kishimoto, ${ }^{b}$ Daisuke Yamaguchi, ${ }^{\mathrm{c}}$ Takayuki Kumada, ${ }^{\mathrm{c}}$ Shin-ichi Takata, ${ }^{\mathrm{d}}$ Kazuki \\ Ohishi $^{\mathbf{e}}$ and Jun-ichi Suzuki ${ }^{\mathrm{e}}$
}

anstitute of Quantum Beam Science, Ibaraki University, Ibaraki, 316-8511, Japan, 'bumitomo Rubber Industries Ltd,
Kobe, 651-0072, Japan, 'Materials Science Research Center, Japan Atomic Energy Agency, Ibaraki, 319-1195, Japan,
dJ-PARC Center, Japan Atomic Energy Agency, Ibaraki, 319-1195, Japan, and ${ }^{\mathbf{e}}$ Neutron Science and Technology Center,
Comprehensive Research Organization for Science and Society (CROSS), Ibaraki, 319-1106, Japan. *Correspondence
e-mail: yohei.noda.77@vc.ibaraki.ac.jp, satoshi.koizumi.prof@vc.ibaraki.ac.jp

Dynamic nuclear polarization (DNP) at low temperature $(1.2 \mathrm{~K})$ and high magnetic field (3.3 T) was applied to a contrast variation study in small-angle neutron scattering (SANS) focusing on industrial rubber materials. By varying the scattering contrast by DNP, time-of-flight SANS profiles were obtained at the pulsed neutron source of the Japan Proton Accelerator Research Complex (J-PARC). The concentration of a small organic molecule, (2,2,6,6-tetramethylpiperidine-1-yl)oxy (TEMPO), was carefully controlled by a doping method using vapour sorption into the rubber specimens. With the assistance of microwave irradiation $(94 \mathrm{GHz})$, almost full polarization of the paramagnetic electronic spin of TEMPO was transferred to the spin state of hydrogen (protons) in the rubber materials to obtain a high proton spin polarization $\left(P_{\mathrm{H}}\right)$. The following samples were prepared: (i) a binary mixture of styrene-butadiene random copolymer (SBR) with silica particles (SBR/SP); and (ii) a ternary mixture of SBR with silica and carbon black particles (SBR/SP/CP). For the binary mixture (SBR/SP), the intensity of SANS significantly increased or decreased while keeping its $q$ dependence for $P_{\mathrm{H}}=-35 \%$ or $P_{\mathrm{H}}=40 \%$, respectively. The $q$ behaviour of SANS for the SBR/SP mixture can be reproduced using the form factor of a spherical particle. The intensity at low $q$ $\left(\sim 0.01 \AA^{-1}\right)$ varied as a quadratic function of $P_{\mathrm{H}}$ and indicated a minimum value at $P_{\mathrm{H}}=30 \%$, which can be explained by the scattering contrast between SP and SBR. The scattering intensity at high $q\left(\sim 0.3 \AA^{-1}\right)$ decreased with increasing $P_{\mathrm{H}}$, which is attributed to the incoherent scattering from hydrogen. For the ternary mixture (SBR/SP/CP), the $q$ behaviour of SANS was varied by changing $P_{\mathrm{H}}$. At $P_{\mathrm{H}}=-35 \%$, the scattering maxima originating from the form factor of SP prevailed, whereas at $P_{\mathrm{H}}=29 \%$ and $P_{\mathrm{H}}=38 \%$, the scattering maxima disappeared. After decomposition of the total SANS according to inverse matrix calculations, the partial scattering functions were obtained. The partial scattering function obtained for SP was well reproduced by a spherical form factor and matched the SANS profile for the SBR/SP mixture. The partial scattering function for $\mathrm{CP}$ exhibited surface fractal behaviour according to $q^{-3.6}$, which is consistent with the results for the SBR/CP mixture.

\section{Introduction}

Contrast variation in small-angle neutron scattering (SANS) is a very useful technique for investigating multi-component systems. For example, in a three-component (ternary) system, contrast variation can emphasize the scattering from a specific component. Therefore, by decomposing the result, we can obtain the partial scattering functions. For this purpose, 
deuterium substitution has conventionally been used, which takes advantage of the difference in neutron scattering length between protons and deuterons. The deuterium substitution technique can easily be applied to solutions or gels, owing to the reasonable availability of deuterated solvents, whereas the synthesis of deuterated polymers is more costly and requires greater effort, especially for industrial materials. Hence, alternative methods applicable to industrial polymer systems are needed.

Besides deuterium substitution, contrast variation can also be achieved by controlling the spin states of both neutrons and protons. The coherent scattering length $\left(b_{\mathrm{coh}, \mathrm{H}}\right)$ and incoherent scattering cross section $\left(\sigma_{\text {inc,H }}\right)$ for hydrogen (or protons) are given by the following equations (Sears, 1992):

$$
b_{\text {coh, } \mathrm{H}}=\left(-0.374+1.456 P_{\mathrm{H}} P_{\mathrm{N}}\right) \times 10^{-12} \mathrm{~cm}
$$

and

$$
\sigma_{\text {inc, } \mathrm{H}}=79.9\left(1-\frac{2}{3} P_{\mathrm{H}} P_{\mathrm{N}}-\frac{1}{3} P_{\mathrm{H}}^{2}\right) \times 10^{-24} \mathrm{~cm}^{2},
$$

where $P_{\mathrm{N}}$ and $P_{\mathrm{H}}$ denote the polarization of neutrons and protons, respectively. Polarization is the difference in populations between up and down spins. Figs. 1(a) and 1(b) show the $P_{\mathrm{H}}$ dependence of $b_{\text {coh,H }}$ and $\sigma_{\text {inc, } \mathrm{H}}$, respectively. Note that the variation in $b_{\mathrm{coh}, \mathrm{H}}$ is about 2.5 times larger than that caused only by deuterium substitution under ordinary conditions (then $P_{\mathrm{H}} P_{\mathrm{N}}=0$ ). For $P_{\mathrm{H}} P_{\mathrm{N}}=0$, the coherent scattering length of hydrogen is -0.374 , whereas that for a deuteron is $0.667 \times$ $10^{-12} \mathrm{~cm}$ (Sears, 1992).

Polarization at thermal equilibrium (TE) states for proton $\left(P_{\mathrm{H}, \mathrm{TE}}\right)$ and electron spins $\left(P_{\mathrm{e}, \mathrm{TE}}\right)$ can be described by

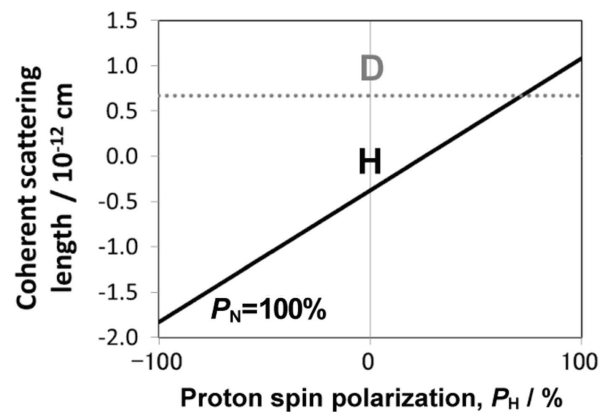

(a)

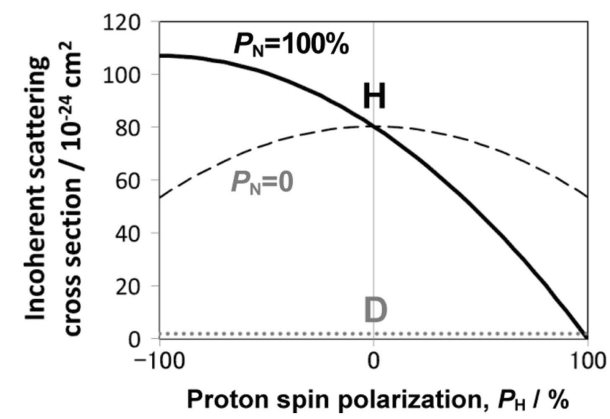

(b)

Figure 1

(a) Neutron coherent scattering length and (b) neutron incoherent scattering cross section of a proton as a function of proton spin polarization $P_{\mathrm{H}}$.
Table 1

Proton and electron spin polarization at thermal equilibrium (3.35 T).

\begin{tabular}{lll}
\hline Temperature & Proton spin polarization & Electron spin polarization \\
\hline $300 \mathrm{~K}$ & $0.001 \%$ & $0.7 \%$ \\
$4.2 \mathrm{~K}$ & $0.082 \%$ & $48 \%$ \\
$1.2 \mathrm{~K}$ & $0.30 \%$ & $95 \%$ \\
\hline
\end{tabular}

considering the Zeeman splitting energy and Boltzmann statistics:

$$
\begin{gathered}
P_{\mathrm{H}, \mathrm{TE}}=\tanh \left(\frac{\hbar \gamma_{\mathrm{H}} H_{0}}{k_{\mathrm{B}} T}\right), \\
P_{\mathrm{e}, \mathrm{TE}}=\tanh \left(\frac{\hbar\left|\gamma_{\mathrm{e}}\right| H_{0}}{k_{\mathrm{B}} T}\right),
\end{gathered}
$$

where $\hbar$ is Planck's constant divided by $2 \pi, \gamma_{\mathrm{H}}$ and $\gamma_{\mathrm{e}}$ are the gyromagnetic ratios of a proton and an electron, respectively, $H_{0}$ is the magnetic field, $k_{\mathrm{B}}$ is Boltzmann's constant, and $T$ is the temperature.

Table 1 lists the polarization values at thermal equilibrium evaluated according to equations (3) and (4). At room temperature, up and down proton spins are almost equally populated. With decreasing temperature, spin polarization increases. However, even at $3.3 \mathrm{~T}$ and $1.2 \mathrm{~K}$, the proton spin is polarized up to only $0.3 \%$. In contrast, the electron spin is polarized up to $95 \%$ under the same conditions (at $3.3 \mathrm{~T}$ and $1.2 \mathrm{~K})$. This is because of the large difference in gyromagnetic ratio between the electron spin and the proton spin $\left(\left|\gamma_{\mathrm{e}}\right| / \gamma_{\mathrm{H}}=\right.$ 658). The large polarization of the electron spin can be transferred to the proton spin by microwave irradiation with energy equal to the simultaneous flipping of electron and proton spins (Abragam \& Goldman, 1978). Consequently, high proton spin polarization is achieved. This is called dynamic nuclear polarization (DNP) and requires electron spin doping, a magnetic field, low temperature and microwave irradiation.

For SANS investigations, proton spin polarization was first applied to structural analysis on the ribosomal protein structure in solution (Stuhrmann et al., 1986). After that pioneering work, several papers reported the utilization of proton spin polarization in neutron scattering experiments (Kohgi et al., 1987; Knop et al., 1992; Fermon et al., 1992; Grinten et al., 1995; Brandt et al., 2006, 2007; Noda et al., 2009, 2011, 2013; Kumada et al., 2010; Stuhrmann, 2015).

Bunyatova (2004) originally developed the vapour sorption technique of TEMPO [(2,2,6,6-tetramethylpiperidine-1-yl)oxy]

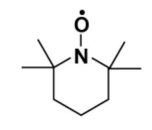

(a)

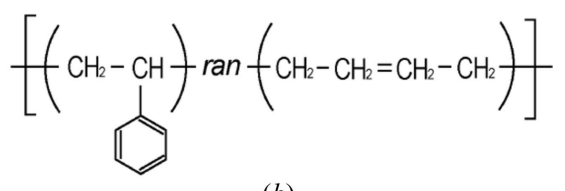

(b)
Figure 2

Chemical structure formulae of (a) TEMPO and (b) styrene-butadiene random copolymer, SBR. 
radicals into solid polymer materials to create polarized targets in nuclear physics experiments. Fig. 2(a) shows the molecular structure formula of TEMPO. On the basis of this technique, we prepared polymer systems for SANS studies after the construction of a DNP cryostat (Kumada et al., $2009 a, b)$ and polarized neutron ultra-small-angle scattering spectrometer (SANS-J-II) (Koizumi et al., 2007) at research reactor JRR-3, Tokai, Japan. We investigated a polyethylene film (Noda et al., 2009) and a di-block copolymer to evaluate precisely the inhomogeneity of the proton polarization around the doped TEMPO molecules (Noda et al., 2011). Subsequently, the vapour sorption technique was successfully applied to silica-filled rubber, which is used for fuel-efficient tyres (Noda et al., 2013). The vapour sorption technique can be applied to industrial rubber products after a manufacturing process.

A tyre, i.e. a multi-component nanocomposite, is an attractive target for contrast variation SANS with DNP. To improve wear and tear resistance, filler particles, such as carbon black (CB) and silica particles (SP), are mixed into the rubber matrix. The spatial distribution of filler particles in the rubber matrix determines not only the tyre's reinforcement but also its energy loss performance (Schaefer et al., 2000; Koga et al., 2005, 2008; Takenaka et al., 2009; Bouty et al., 2014; Genix \& Oberdisse, 2015). In accordance with the empirical knowledge that a homogeneous dispersion of filler particles lowers the energy loss, various attempts towards dispersion control have been conducted (Byers, 2002). To optimize tyre rubber performance, a reliable methodology for evaluating the filler particle dispersion is critical.

The combination of $\mathrm{CB}$ and SP is frequently used for manufacturing tyres. In addition to the above-mentioned effects, CB is advantageous for specific UV resistance and electric discharge. For precise structural analyses, we need to decompose the total SANS observed for a multi-component system into individual partial scattering functions.

In this article, we report our recent achievements on DNP and contrast variation SANS on model mixtures for industrial tyres. At the Materials and Life Science Experimental Facility (MLF) of the Japan Proton Accelerator Research Complex (J-PARC), we performed time-of-flight (TOF) SANS experiments, employing a wide range of neutron wavelength $(\lambda)$. This causes imperfect neutron polarization $\left(P_{\mathrm{N}}\right)$, depending on $\lambda$ and variations in the coherent and incoherent scattering lengths.

\section{Experimental}

\subsection{Sample preparation}

As a model system for an industrial tyre, we prepared two types of rubber specimen: a binary mixture of styrene-butadiene random copolymer (SBR) with silica particles (SBR/ $\mathrm{SP}$ ), and a ternary mixture of SBR with silica and CB particles (SBR/SP/CP). Fig. 2(b) shows the molecular structure formula of SBR. As listed in Table 2, the samples consist of solutionSBR (S-SBR, Buna VSL 4720, Lanxess Corp.), silica particles
Table 2

Specimen composition in vol.\%.

DCP is dicumyl peroxide, TBBS is $N$-tert-butyl-2-benzothiazyl sulfenamide, DPG is 1,3-diphenylguanidine and Acc. denotes accelerator.

\begin{tabular}{|c|c|c|c|c|c|c|c|c|}
\hline Specimen & S-SBR & Silica & $\mathrm{CB}$ & $\begin{array}{l}\text { Stearic } \\
\text { acid }\end{array}$ & $\begin{array}{l}\text { Silane-coupling } \\
\text { agent }\end{array}$ & DCP & $\begin{array}{l}\text { Acc. } \\
\text { TBBS }\end{array}$ & $\begin{array}{l}\text { Acc. } \\
\text { DPG }\end{array}$ \\
\hline SBR/SP & 84.6 & 10.0 & 0.00 & 1.87 & 1.49 & 0.71 & 0.62 & 0.66 \\
\hline $\mathrm{SBR} / \mathrm{SP} / \mathrm{CP}$ & 82.7 & 10.0 & 2.00 & 1.83 & 1.50 & 0.69 & 0.60 & 0.65 \\
\hline
\end{tabular}

(Seahostar KE-P10, Nippon Shokubai Co. Ltd), CB (N330, Tokai Carbon Co. Ltd), silane coupling agent (Si 69, Evonik Degussa $\mathrm{GmbH}$ ) and other additives. We selected silica particles with a narrow radius distribution.

All ingredients were mixed in a milling machine and the resulting mixture was pressed into a mould and kept at $443 \mathrm{~K}$ for $20 \mathrm{~min}$. The thicknesses of the SBR/SP and SBR/SP/CP specimens produced were 0.56 and $0.24 \mathrm{~mm}$, respectively.

\subsection{Vapour absorption of TEMPO into the rubber}

In order to perform DNP, an electron spin source is required in a specimen. By a vapour sorption technique, the stable free radical molecule TEMPO (Fig. 2a) was introduced into the rubbery mixture prepared in $\$ 2.1$. The vaporized TEMPO radicals were spontaneously absorbed and diffused into the amorphous matrix of SBR. We placed the rubber

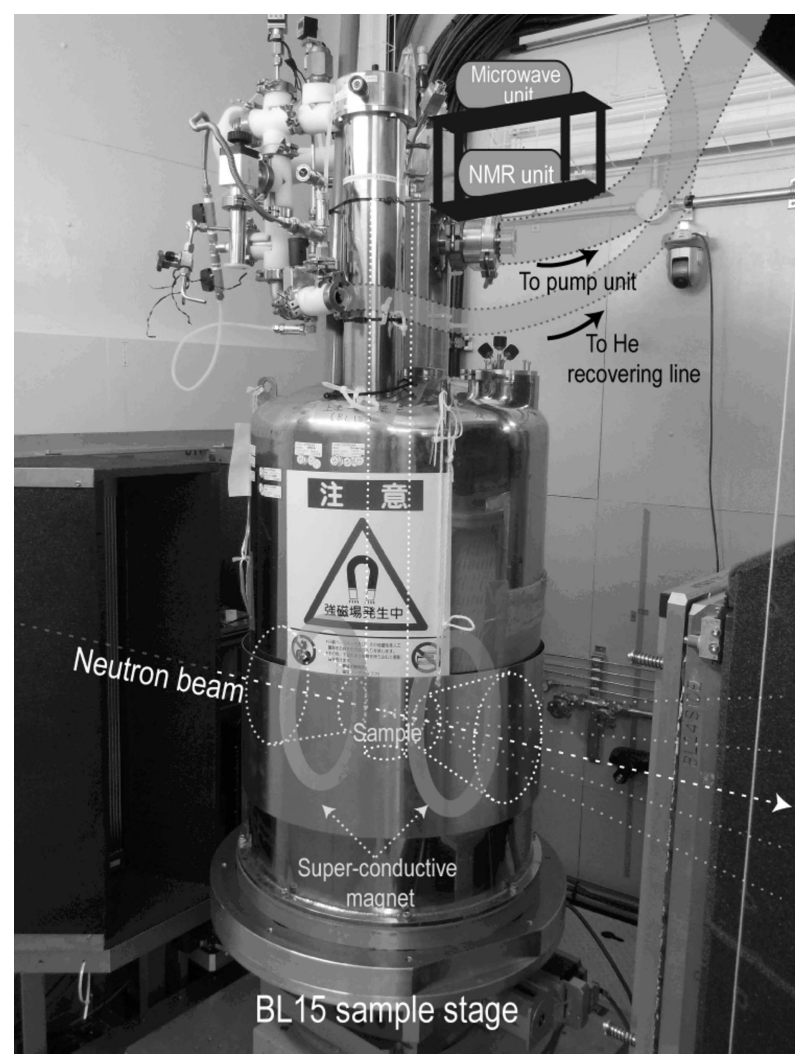

Figure 3

A photograph of the DNP cryostat. 

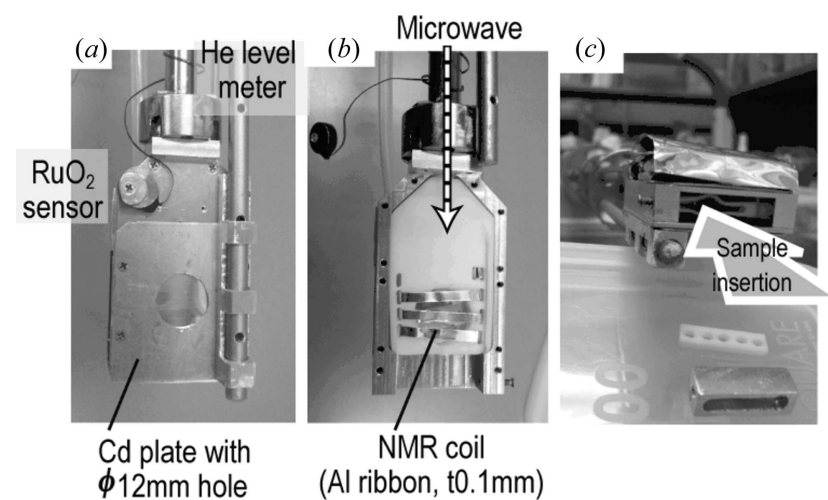

Figure 4

The sample cell of the DNP cryostat. Further detail for parts $(a)$ to $(c)$ is given in the text.

mixtures with TEMPO inside a sealed container at $313 \mathrm{~K}$ for 1 week. Consequently, the vaporized TEMPO spontaneously permeated the rubber matrix. By electron spin resonance measurements, the TEMPO concentrations were determined at 37 and $35 \mathrm{~m} M$ for the binary (SBR/SP) and ternary (SBR/ $\mathrm{SP} / \mathrm{CP}$ ) mixtures, respectively. These concentrations were close enough to the optimum value $(30 \mathrm{mM})$ for DNP.

The influence of the added TEMPO on the microstructure should be noted. We confirmed experimentally that SANS obtained for the mixture after TEMPO doping coincided with that obtained for the mixture before doping. The static structure, which is a target of this contrast variation SANS study, was not affected by the addition of TEMPO. On the other hand, regarding dynamic properties, the storage and loss modulus (Busfield et al., 2000) and the longitudinal and transverse proton relaxation times (Stapf \& Kariyo, 2005)
Table 3

Specification of the DNP cryostat.

\begin{tabular}{ll}
\hline Magnetic field & $\begin{array}{c}3.35 \mathrm{~T} \text {, parallel with neutron beam, } \\
\text { inhomogeneity }<0.5 \times 10^{-4} \text { (relative) }\end{array}$ \\
Specimen temperature & $1.2 \mathrm{~K}$ (single-shot) ${ }^{4} \mathrm{He}$ evaporation cryostat \\
Microwave & $94 \mathrm{GHz}$ Gunn oscillator \\
NMR circuit & $144 \mathrm{MHz}$, continuous-wave, frequency-sweep
\end{tabular}

were affected by the addition of small organic molecules (a few per cent in weight), which is known as the 'plasticizer' effect.

\subsection{DNP}

Fig. 3 shows the DNP cryostat used in this study (Kumada et $a l ., 2009 a, b)$. A rubber specimen is placed in a chamber filled with liquid ${ }^{4} \mathrm{He}$. By evaporating the liquid ${ }^{4} \mathrm{He}$, the specimen is cooled to $1.2 \mathrm{~K}$ during continuous microwave irradiation $(94 \mathrm{GHz})$. The sample chamber is located between the splittype superconducting magnet coils. The superconducting magnet generates a magnetic field up to $3.5 \mathrm{~T}$ at the sample position. The magnetic field is parallel to the neutron beam direction. The inhomogeneity of the magnetic field $\left(\Delta B / B_{0}\right)$ was designed to be less than $10^{-4}$ to avoid proton spin depolarization. The neutron beam passes along the central axis of the magnet coils. The windows through which the neutron beam passes are formed of thin aluminium plates, which cause less background scattering.

Fig. 4(a) shows a photograph of the sample cell. On the upstream side, a Cd plate with a $12 \mathrm{~mm}$ diameter hole is fixed. A thin $(0.1 \mathrm{~mm})$ aluminium sheet is fixed inside to prevent microwave leakage. The aluminium case has several holes for

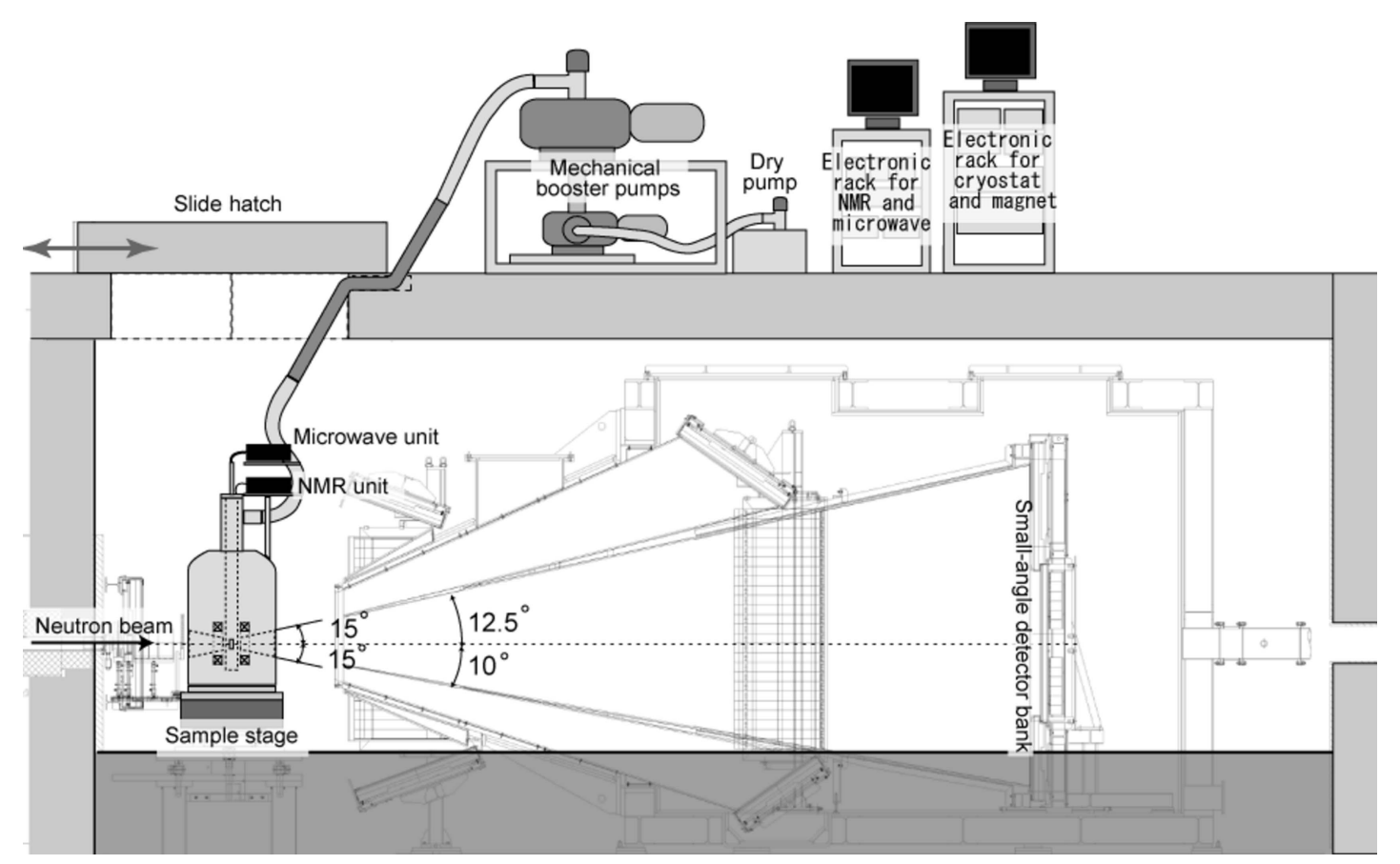

Figure 5

The DNP experimental layout on BL15 TAIKAN of the MLF at J-PARC. 
circulating liquid $\mathrm{He}$; the diameter of these holes is $0.5 \mathrm{~mm}$, which is much less than the $94 \mathrm{GHz}$ microwave wavelength $(3 \mathrm{~mm})$. Fig. 4(b) shows a photograph of the sample cell when the upstream side cover is removed. Inside the aluminium case, a polytetrafluoroethylene (PTFE) part supports a threeturn NMR coil made of $0.1 \mathrm{~mm}$-thick aluminium plate for $P_{\mathrm{H}}$ evaluation. $P_{\mathrm{H}}$ is evaluated using the continuous-wave NMR circuit, which is described later in \$3.1. As shown in Fig. 4(c), a sheet sample $(14 \times 14 \times 1 \mathrm{~mm})$ is inserted from the bottom into the NMR coil. After insertion of the sample, the aluminium block supporting the sample bottom is fixed by screws. Microwave radiation $(94 \mathrm{GHz})$ is generated by the Gunn oscillator fixed over the top plate of the cryostat, which irradiates the sample through a stainless steel pipe of length $1 \mathrm{~m}$ and inside diameter $6 \mathrm{~mm}$, filled with a PTFE rod. The specifications of the DNP cryostat are summarized in Table 3.

\subsection{SANS}

TOF-SANS experiments were performed on the TAIKAN instrument (BL15) (Shinohara, Suzuki et al., 2009; Shinohara, Takata et al., 2009; Takata et al., 2015) at the MLF of J-PARC. The TAIKAN instrument is equipped with a magnetic supermirror polarizer, composed of an $\mathrm{Fe} / \mathrm{Si}$ multilayer $\left(4 Q_{\mathrm{c}}\right)$. Fig. 5 shows a schematic diagram of the device allocations on the SANS instrument. The roof of the shielding room has a sliding hatch covering the sample stage. Through this sliding hatch, the DNP cryostat was introduced onto the sample stage. The DNP cryostat was originally designed for SANS-J-II at JRR-3 and cannot fully cover the detectors of TAIKAN, having detectors for neutrons with wider scattering angles $(2 \theta$ $>15^{\circ}$ ). The roof of the shielding room has two trenches under the sliding hatch. Through these trenches, pipes and cables were pulled out. The pipes are necessary to evaporate liquid

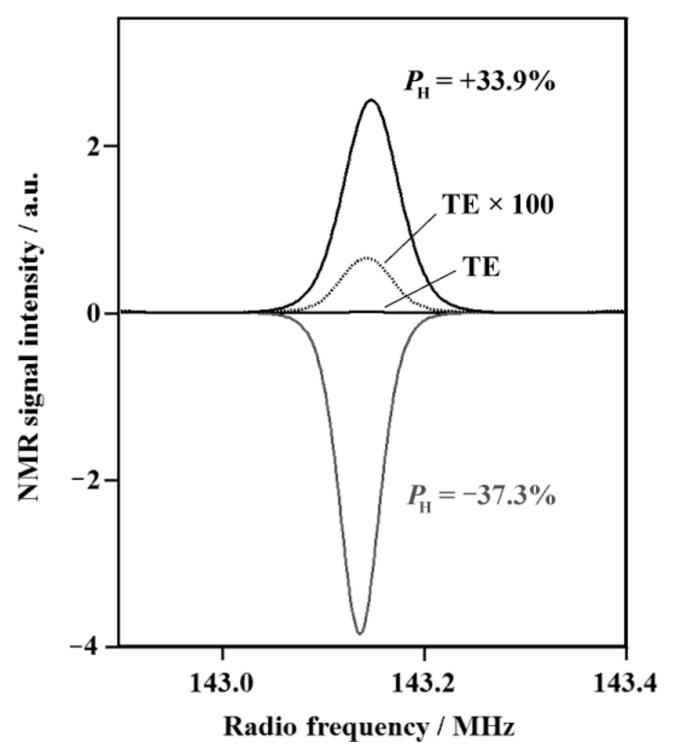

Figure 6

Proton NMR signal enhancement by DNP. TE denotes the signal observed at a thermal equilibrium of $3.35 \mathrm{~T}$ and $4.2 \mathrm{~K}\left(P_{\mathrm{H}}=0.082 \%\right)$. The sample was a $25 \mathrm{~m} M$ TEMPO-doped SBR rubber without filler.
${ }^{4} \mathrm{He}$ and to supply ${ }^{4} \mathrm{He}$ gas. The cables are for energizing the superconducting magnet, monitoring the signals from the liquid helium level meter and the pressure and temperature sensors, measuring the NMR signals, and supplying voltage to the microwave generator. The mechanical booster pump unit and the two electronic racks were placed on the roof of the shield room. Consequently, we remotely controlled the magnetic field, sample temperature, NMR measurements and microwave irradiation.

\section{Experimental results and discussion}

\subsection{Proton NMR}

Prior to SANS experiments with proton spin polarization, it is important to investigate the proton spin polarization behaviour of the TEMPO-doped rubber specimens using proton NMR. Fig. 6 shows the results of proton NMR. The integrated NMR signal was calibrated in order to coincide with the thermal equilibrium $P_{\mathrm{H}}=0.082 \%$ at $3.35 \mathrm{~T}$ and $4.2 \mathrm{~K}$, which is given according to equation (3). Then by NMR, we can evaluate the bulk $P_{\mathrm{H}}$ averaged over a whole specimen.

Fig. 7 shows the bulk $P_{\mathrm{H}}$ as a function of microwave frequency. By tuning the microwave frequency, the bulk $P_{\mathrm{H}}$ is converted from positive to negative. The microwave frequency can easily be controlled by the voltage supplied to the Gunn oscillator. The bulk $P_{\mathrm{H}}$ also depends not only on the microwave frequency but also on the sample temperature.

Fig. 8 shows the bulk $P_{\mathrm{H}}$ determined as a function of TEMPO concentration. At $30 \mathrm{~m} M,\left|P_{\mathrm{H}}\right|$ reaches a maximum value. Fig. 8 also shows the proton spin relaxation time. As the TEMPO concentration increases, the spin relaxation time decreases monotonically owing to the increase in magnetic field fluctuation which is caused by TEMPO radicals.

Fig. 9 shows the time profile of the bulk $P_{\mathrm{H}}$ during the SANS experiment on the binary mixture (SBR/SP). Each SANS measurement was performed when the bulk $P_{\mathrm{H}}$ was kept constant, as indicated by the shaded areas in Fig. 9. From 1.8 to $2.2 \mathrm{~h}$, the bulk $P_{\mathrm{H}}$ was swept in order to search for the contrast matching condition by changing the microwave

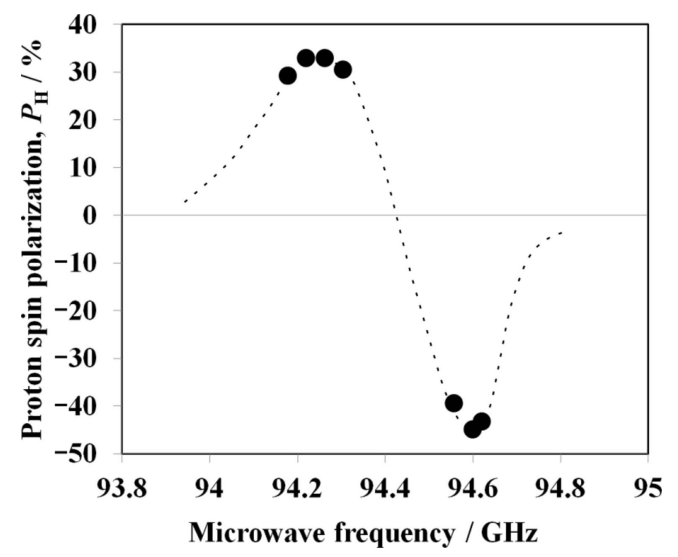

Figure 7

The microwave frequency dependence of proton spin polarization. The sample was a $25 \mathrm{mM}$ TEMPO-doped SBR rubber without filler. 


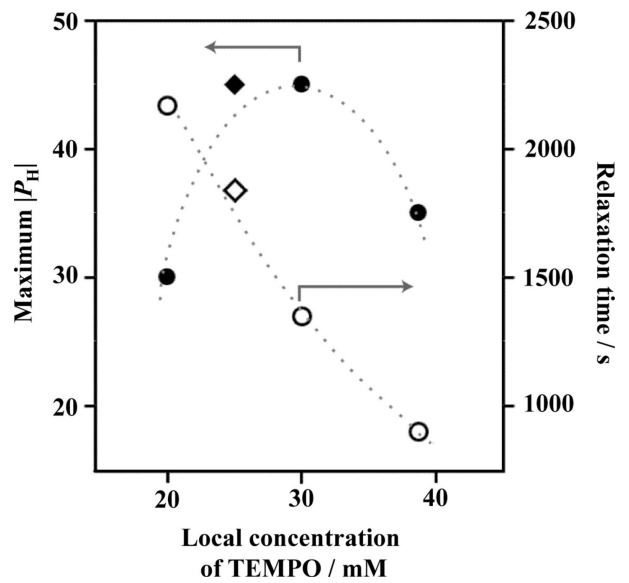

Figure 8

The maximum achievable $\left|P_{\mathrm{H}}\right|$ for silica-filled SBR rubber samples as a function of local concentration of TEMPO.

frequency. The bulk $P_{\mathrm{H}}$ quickly responded to the microwave frequency tuning (the time constant was 150-200 s). The fast response of $P_{\mathrm{H}}$ is convenient for SANS experiments.

In the time region with no shading, the bulk $P_{\mathrm{H}}$ changed continuously. Therefore, the coherent scattering length, and in turn the scattering contrast, also varied continuously. For such dynamic behaviours, the event-data format, which is commonly used in TOF-SANS experiments, is advantageous for extracting data from favourable time domains after the experiment.

\subsection{SANS for the binary mixture (SBR/SP)}

3.2.1. SANS without polarization. Fig. $10(a)$ shows SANS obtained for the binary mixture (SBR/SP) without polarizing proton spins $\left(P_{\mathrm{H}}=0 \%\right)$. The SANS intensity is shown as a function of the magnitude of the scattering vector $q[q=(4 \pi /$ $\lambda) \sin (\theta / 2)$, where $\lambda$ and $\theta$ are the wavelength of the neutrons and the scattering angle, respectively]. In the low- $q$ region, scattering maxima originating from the form factor of the SPs were observed. The scattering curves (thick grey curves in

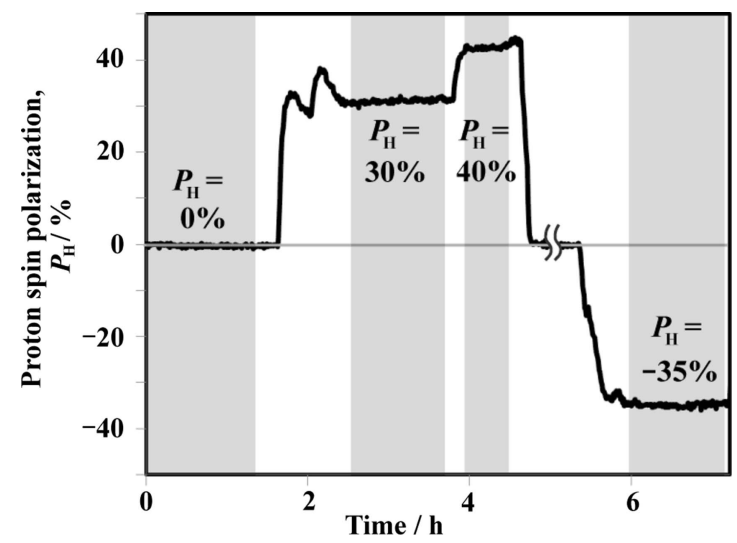

Figure 9

The time evolution of $P_{\mathrm{H}}$ evaluated by NMR during the SANS experiment on SBR/SP. The SANS measurements were performed during the periods shown in grey.
Figs. $10 a$ and $10 b$ ) were reproduced using equations (5)-(7) and considering a smearing effect for the BL15 collimation geometry:

$$
I(q)=\int \frac{f_{\mathrm{v}}}{\left(\frac{4}{3} \pi R^{3}\right)} W(R) F(q ; R)^{2} \mathrm{~d} R
$$

where

$$
F(q ; R)=\Delta \rho\left(\frac{4}{3} \pi R^{3}\right) \frac{3(\sin q R-q R \cos q R)}{(q R)^{3}}
$$

and

$$
W(R)=\frac{1}{\left(2 \pi \sigma^{2}\right)^{1 / 2}} \exp \left[-\frac{\left(R-R_{0}\right)^{2}}{2 \sigma^{2}}\right] .
$$

In equation (5), $f_{\mathrm{v}}$ is the volume fraction of spherical particles. In equation (6), $F(q ; R)$ is the scattering amplitude for a spherical particle of radius $R$, and $\Delta \rho$ is the scattering length density difference between the spherical particles and the surrounding medium. $W(R)$ in equation (7) is a Gaussian function for the radius distribution, where $R_{0}$ is the average radius and $\sigma$ is the radius dispersion. As a result of our evaluations, we obtained $R_{0}=610 \AA$ and $\sigma=40 \AA$. At that time, we calibrated SANS on the absolute intensity scale $\left(\mathrm{cm}^{-1}\right)$ using the known volume fraction of SP.

3.2.2. SANS under polarization. As seen in Fig. 10, the intensity of SANS at low $q$ increased at negative polarization $P_{\mathrm{H}}=-35 \%$. For positive polarization, the scattering intensity decreased at $P_{\mathrm{H}}=30 \%$ and increased again at $P_{\mathrm{H}}=40 \%$. In order to examine the scattering intensity depending on the bulk $P_{\mathrm{H}}$, we evaluated the coherent scattering length density of SBR according to equation (1) and the chemical composition of SBR. Simultaneously, we separately evaluated the coherent scattering length density for polystyrene (PS) and polybutadiene (PB) monomers, which compose the SBR chain. Note that the SANS scattering intensity is proportional to the square of the difference in the coherent scattering

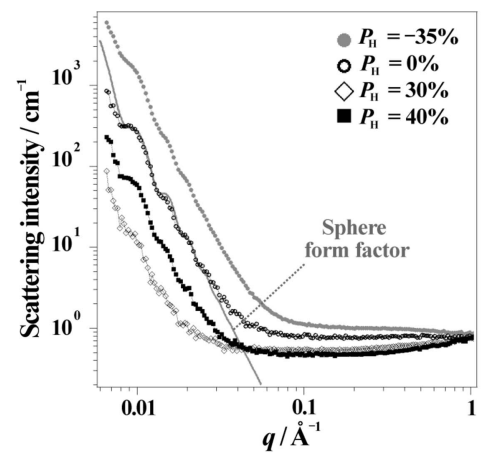

(a)

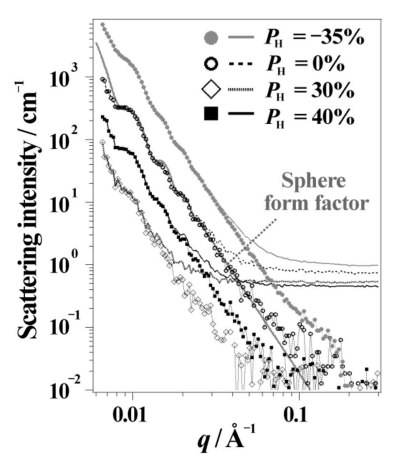

(b)
Figure 10

The SANS intensity of SBR/SP. In the profiles in panel $(a)$, neutrons in the full wavelength range $(1<\lambda<7.6 \AA)$ were used. In the profiles in panel $(b)$, neutrons in a limited wavelength range $(4<\lambda<7.6 \AA)$ were used. In panel $(b)$, the symbols represent the profiles after subtracting the incoherent scattering. The thick grey profiles in panels $(a)$ and $(b)$ were calculated using the spherical form factor with radius $610 \pm 40 \AA$ 
length density. We found that the matching point between silica and SBR appears at $P_{\mathrm{H}}=30 \%$ (Fig. 11a).

As shown in Fig. 10(a), at high $q$ between 0.1 and $0.3 \AA^{-1}$, the SANS exhibited $q$-independent behaviour. It can readily be seen that the scattering intensity in this $q$ region has decreased with increasing $P_{\mathrm{H}}$. This is consistent with the description for incoherent scattering according to equation (2). However, the influence of the imperfect neutron polarization on the incoherent scattering should be noted. If we postulate $P_{\mathrm{N}}=0$ in equation (2), we obtain

$$
\sigma_{\text {inc }, \mathrm{H}}=79.9\left(1-\frac{1}{3} P_{\mathrm{H}}^{2}\right) \times 10^{-24} \mathrm{~cm}^{2} .
$$

In Fig. $1(b), \sigma_{\text {inc, } \mathrm{H}}$ with $P_{\mathrm{N}}=0$ is shown by the dashed line, indicating clearly different behaviour from the case with $P_{\mathrm{N}}=1$.

In the TOF-SANS experiments, we utilized neutrons with a wide $\lambda$ range simultaneously. The shorter- $\lambda$ neutrons contribute to the higher- $q$ region, whereas the longer- $\lambda$ neutrons contribute to the lower- $q$ region. Fig. 12 shows the $P_{\mathrm{N}}$ provided at TAIKAN as a function of $\lambda . P_{\mathrm{N}}$ has decreased with decreasing $\lambda$ for $\lambda<4 \AA$, and become almost zero for $\lambda<1 \AA$.

Polarized neutrons are used effectively in polarization analysis for separating coherent and incoherent scattering. In this technique, by examining the spin-flip and non-spin-flip contributions, we can separate the coherent and incoherent scattering contributions. In the separation process, the $\lambda$ dependence of $P_{\mathrm{N}}$ can easily be corrected.

However, in the case of spin contrast variation, the $\lambda$ dependence of $P_{\mathrm{N}}$ affects not only the incoherent scattering length but also the coherent scattering length. Therefore, in the following analysis in this study, we only use data with $4<$ $\lambda<7.6 \AA$, where $P_{\mathrm{N}}>97 \%$ is satisfied. The results are shown in Fig. $10(b)$. The observed $q$ region is limited $\left(q<0.3 \AA^{-1}\right)$ because we excluded the shorter- $\lambda$ contribution.

3.2.3. Transmission under polarization. The transmission (T) increased with increasing $P_{\mathrm{H}}$, as shown in Fig. 13(a). $T$ is given by

$$
T=\exp \left(-\Sigma_{\text {tot }} t\right)
$$

where $t$ is the sample thickness and $\Sigma_{\text {tot }}$ is given by

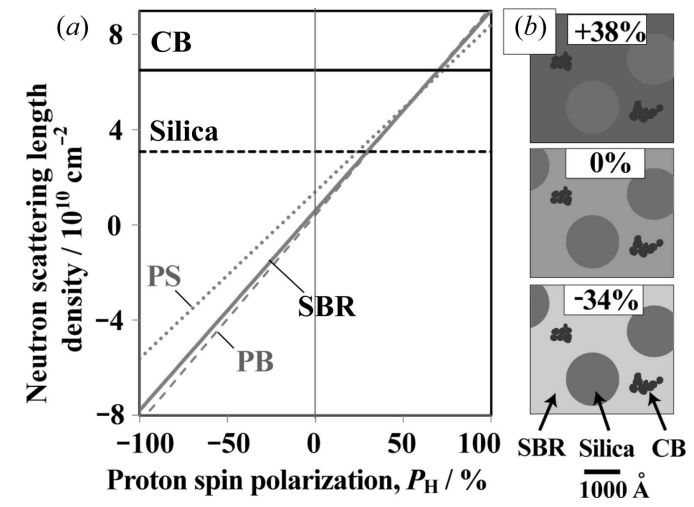

Figure 11

(a) Neutron scattering length density as a function of $P_{\mathrm{H}}$. (b) Schematics of the microstructure of SBR/SP/CP. The degree of shading reflects the neutron scattering length density.

$$
\Sigma_{\mathrm{tot}}=\sum_{i} n_{i} \sigma_{\mathrm{tot}, i}
$$

Here, $i$ is the index for labelling the nuclear species, $n_{i}$ is the number density for the labelled nuclear species and $\sigma_{\text {tot }, i}$ is the total cross section (the sum of coherent scattering, incoherent scattering and absorption cross sections) of the labelled nuclear species. For protons, the total scattering cross section $\left(\sigma_{\text {tot,H }}\right)$ is given by

$$
\sigma_{\text {tot }, \mathrm{H}}=\left(81.99-66.97 P_{\mathrm{H}}\right) \times 10^{-24} \mathrm{~cm}^{2},
$$

and for SBR/SP, $\Sigma_{\mathrm{tot}}=4.49-3.48 P_{\mathrm{H}} \mathrm{cm}^{-1}$. In Fig. 13(a), $T$ calculated by equation (9) is indicated by the dotted line. The transmission determined experimentally is consistent with the evaluation by equation (9), although the values are slightly high. The difference might be attributed to sample thickness distribution or multiple and inelastic scattering.

3.2.4. In the low- $q$ region. The scattering intensity at low $q$ ( $q=0.01 \AA^{-1}$ ) is shown as a function of $P_{\mathrm{H}}$ in Fig. 13(b). In the low- $q$ region, the coherent scattering originating from silica particles is dominant. The solid line in Fig. 13(b) exhibits a contrast factor in between those of the silica and SBR phases $\left[\left(\rho_{\mathrm{SP}}-\rho_{\mathrm{SBR}}\right)^{2}\right]$, where $\rho_{\mathrm{SP}}$ is the scattering length density of silica $\left(3.08 \times 10^{10} \mathrm{~cm}^{-2}\right)$ and $\rho_{\mathrm{SBR}}$ is the scattering length density of SBR $\left[\left(0.62+8.39 P_{\mathrm{H}}\right) \times 10^{10} \mathrm{~cm}^{-2}\right]$. The evaluated solid line agrees well with the experimental results.

3.2.5. In the high- $q$ scattering region. The scattering intensity at high $q\left(q=0.3 \AA^{-1}\right)$ is shown as a function of $P_{\mathrm{H}}$ in Fig. 13(c). In this high- $q$ region, incoherent scattering $\left(I_{\text {inc }}\right)$ is dominant because the coherent scattering intensity from silica particles decreases drastically with increasing $q . I_{\text {inc }}$ is given by

$$
I_{\mathrm{inc}}=\frac{\Sigma_{\mathrm{inc}}}{4 \pi},
$$

where $\Sigma_{\text {inc }}$ is the sum of the incoherent scattering cross section per unit volume,

$$
\Sigma_{\mathrm{inc}}=\sum_{i} n_{i} \sigma_{\mathrm{inc}, i}
$$

Here, $i$ is the index for labelling nuclear species, $n_{i}$ is the number density for the labelled nuclear species and $\sigma_{\text {inc }, i}$ is the incoherent scattering cross section of the labelled nuclear species. Using $\sigma_{\mathrm{inc}, \mathrm{H}}$ in equation (2) for SBR/SP, equation (13) transforms to

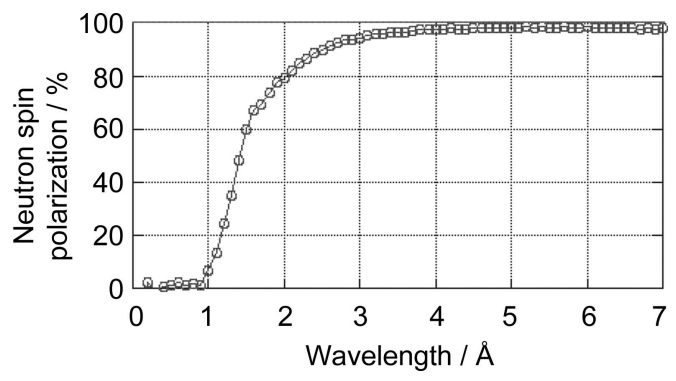

Figure 12

Neutron spin polarization as a function of neutron wavelength, $\lambda$, obtained for the BL15 supermirror polarizer. 


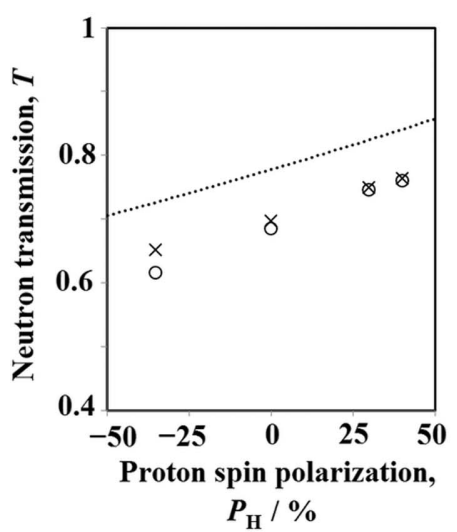

(a)

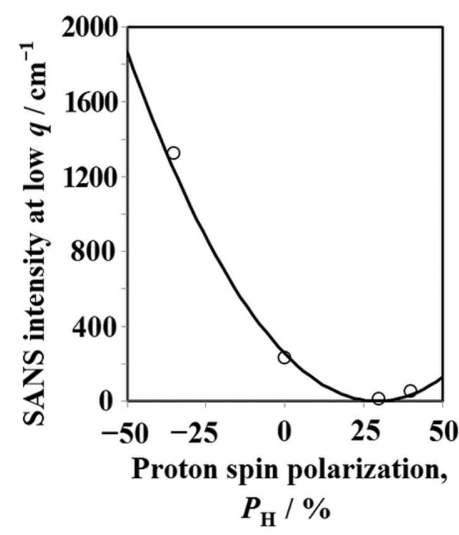

(b)

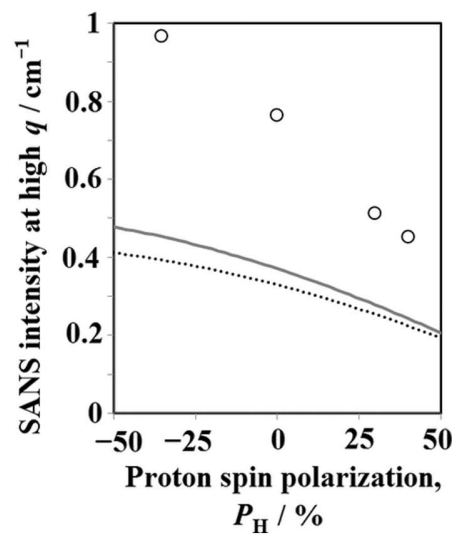

(c)

Figure 13

(a) Neutron transmission, $(b)$ scattering intensity at low $q\left(0.01 \AA^{-1}\right)$ and $(c)$ scattering intensity at high $q\left(0.3 \AA^{-1}\right)$, all as a function of $P_{\mathrm{H}}$ for SBR/SP. In panel $(a)$, the circles and crosses denote the results for neutrons with $\lambda=6 \AA$ and $\lambda=4 \AA$, respectively. The dotted line is the calculated exp $\left(-\Sigma_{\text {tot }} t\right)$. In panel $(c)$, the dotted and solid grey lines are the calculated $\Sigma_{\text {ind }} d(4 \pi)$ and $\left[\exp \left(\Sigma_{\text {inc }} t\right)-1\right] /(4 \pi t)$, respectively.

$$
\Sigma_{\text {inc }}=4.14\left(1-\frac{2}{3} P_{\mathrm{H}}-\frac{1}{3} P_{\mathrm{H}}^{2}\right) \mathrm{cm}^{-1} .
$$

The calculated results are shown in Fig. $13 c$ by the dotted line, which does not reproduce the experiments well. By considering multiple scattering effects, $I_{\text {inc }}$ is given by the following equation (Shibayama et al., 2005):

$$
I_{\text {inc }}=\frac{\exp \left(\Sigma_{\text {inc }} t\right)-1}{4 \pi t} .
$$

In Fig. 13(c), the solid grey line is calculated using equation (15) and a thickness $t=0.056 \mathrm{~cm}$. It is closer to the experimental result, but still lower. This discrepancy might be attributed to the coherent scattering contribution that still exists in the high- $q$ region. As shown in Fig. 11, the coherent scattering length densities of the PS and PB composing SBR deviate from each other for negative $P_{\mathrm{H}}$. Thus, local concentration fluctuations between PS and PB might give rise to coherent scattering, even at high $q$.

\subsection{SANS for the ternary mixture $(\mathrm{SBR} / \mathrm{SP} / \mathrm{CP})$}

Fig. 14(a) shows the SANS results obtained for the ternary mixture system (SBR/SP/CP). According to the discussion in $\$ 3.2$ for the data reduction, we employed the limited wavelength range of $4<\lambda<7.6 \AA$, which gives $P_{\mathrm{N}}>97 \%$. As expected for a ternary mixture, the $q$ dependence of the SANS varies significantly with changing proton spin polarization; the scattering maxima due to silica particles were observed for $P_{\mathrm{H}}=0 \%$ and $P_{\mathrm{H}}=-34 \%$, whereas they disappeared for $P_{\mathrm{H}}=$ $29 \%$ and $P_{\mathrm{H}}=38 \%$. As already shown in Fig. 11, the scattering length densities of silica and SBR match at $P_{\mathrm{H}}=30 \%$. Around the matching point, the $\mathrm{CB}$ contribution was observed more clearly.

3.3.1. Partial scattering function decomposition. The SANS intensity for the ternary mixture is given by the sum of three partial scattering functions $\left[S_{\mathrm{SP}-\mathrm{SP}}(q), S_{\mathrm{CP}-\mathrm{CP}}(q)\right.$ and $\left.S_{\mathrm{SP}-\mathrm{CP}}(q)\right]$ as follows:

$$
\begin{aligned}
& I\left(q ; P_{\mathrm{H}}\right)=\left[\rho_{\mathrm{SP}}-\rho_{\mathrm{SBR}}\left(P_{\mathrm{H}}\right)\right]^{2} S_{\mathrm{SP}-\mathrm{SP}}(q) \\
& \quad+\left[\rho_{\mathrm{CP}}-\rho_{\mathrm{SBR}}\left(P_{\mathrm{H}}\right)\right]^{2} S_{\mathrm{CP}-\mathrm{CP}}(q) \\
& \quad+2\left[\rho_{\mathrm{SP}}-\rho_{\mathrm{SBR}}\left(P_{\mathrm{H}}\right)\right]\left[\rho_{\mathrm{CP}}-\rho_{\mathrm{SBR}}\left(P_{\mathrm{H}}\right)\right] S_{\mathrm{SP}-\mathrm{CP}}(q),
\end{aligned}
$$

where the partial scattering functions are weighted by a contrast factor. In this equation, $\rho_{\mathrm{SP}}, \rho_{\mathrm{CP}}$ and $\rho_{\mathrm{SBR}}$ correspond to the neutron scattering length densities of the silica, $\mathrm{CB}$ and SBR phases, respectively: $\rho_{\mathrm{SP}}=3.08 \times 10^{10} \mathrm{~cm}^{-2}, \rho_{\mathrm{CP}}=6.50 \times$ $10^{10} \mathrm{~cm}^{-2}$ and $\rho_{\mathrm{SBR}}=\left(0.62+8.39 P_{\mathrm{H}}\right) \times 10^{10} \mathrm{~cm}^{-2}$. Only $\rho_{\mathrm{SBR}}$ depends on $P_{\mathrm{H}}$, because SBR contains hydrogen. $S_{\mathrm{SP}-\mathrm{SP}}(q)$, $S_{\mathrm{CP}-\mathrm{CP}}(q)$ and $S_{\mathrm{SP}-\mathrm{CP}}(q)$ are defined by the following equation:

$$
S_{i-j}(\mathbf{q})=\frac{1}{V} \iint \delta \varphi_{i}(\mathbf{r}) \delta \varphi_{j}\left(\mathbf{r}^{\prime}\right) \exp \left[-i \mathbf{q} \cdot\left(\mathbf{r}-\mathbf{r}^{\prime}\right)\right] \mathrm{d} \mathbf{r} \mathrm{d} \mathbf{r}^{\prime},
$$

where the subscript $i$ or $j$ labels one of the components (SP, CP or SBR) and $\delta \varphi_{i}(\mathbf{r})$ indicates the fluctuation in the volume fraction of component $i$ at position $\mathbf{r}$. We observed the SANS

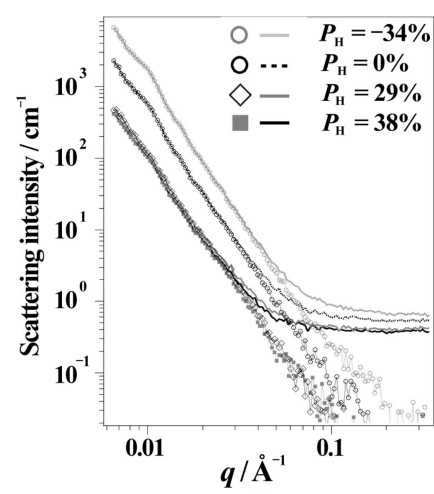

(a)

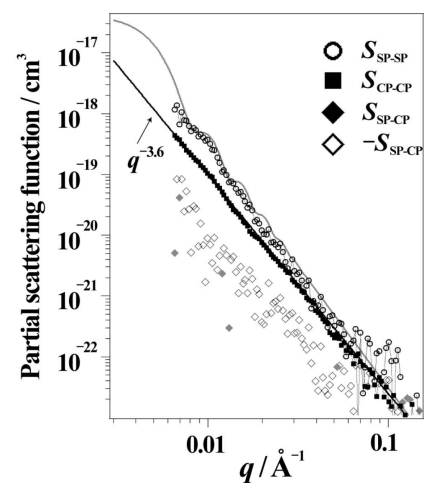

(b)

\section{Figure 14}

(a) The SANS profiles for SBR/SP/CP. The scattering profiles after subtraction of the incoherent scattering are denoted by symbols. (b) The separated partial scattering functions $\left(S_{\mathrm{SP}-\mathrm{SP}}, S_{\mathrm{CP}-\mathrm{CP}}\right.$ and $\left.S_{\mathrm{SP}-\mathrm{CP}}\right)$. The grey curve is the calculated spherical form factor with the parameters determined for SBR/SP. 
at four different $P_{\mathrm{H}}$ (Fig. 14a). Then, the $I\left(q ; P_{\mathrm{H}, i}\right)$ for different $P_{\mathrm{H}, i}(i=1$ to 4$)$ are described by

$$
\begin{aligned}
& {\left[\begin{array}{c}
I\left(q ; P_{\mathrm{H}, 1}\right) \\
I\left(q ; P_{\mathrm{H}, 2}\right) \\
I\left(q ; P_{\mathrm{H}, 3}\right) \\
I\left(q ; P_{\mathrm{H}, 4}\right)
\end{array}\right]=} {\left[\begin{array}{lll}
\Delta_{\mathrm{SP}-\mathrm{SP}}\left(P_{\mathrm{H}, 1}\right) & \Delta_{\mathrm{CP}-\mathrm{CP}}\left(P_{\mathrm{H}, 1}\right) & \Delta_{\mathrm{SP}-\mathrm{CP}}\left(P_{\mathrm{H}, 1}\right) \\
\Delta_{\mathrm{SP}-\mathrm{SP}}\left(P_{\mathrm{H}, 2}\right) & \Delta_{\mathrm{CP}-\mathrm{CP}}\left(P_{\mathrm{H}, 2}\right) & \Delta_{\mathrm{SP}-\mathrm{CP}}\left(P_{\mathrm{H}, 2}\right) \\
\Delta_{\mathrm{SP}-\mathrm{SP}}\left(P_{\mathrm{H}, 3}\right) & \Delta_{\mathrm{CP}-\mathrm{CP}}\left(P_{\mathrm{H}, 3}\right) & \Delta_{\mathrm{SP}-\mathrm{CP}}\left(P_{\mathrm{H}, 3}\right) \\
\Delta_{\mathrm{SP}-\mathrm{SP}}\left(P_{\mathrm{H}, 4}\right) & \Delta_{\mathrm{CP}-\mathrm{CP}}\left(P_{\mathrm{H}, 4}\right) & \Delta_{\mathrm{SP}-\mathrm{CP}}\left(P_{\mathrm{H}, 4}\right)
\end{array}\right] } \\
& \times\left[\begin{array}{c}
S_{\mathrm{SP}-\mathrm{SP}}(q) \\
S_{\mathrm{CP}-\mathrm{CP}}(q) \\
S_{\mathrm{SP}-\mathrm{CP}}(q)
\end{array}\right] \\
& \equiv \mathbf{M}\left[\begin{array}{c}
S_{\mathrm{SP}-\mathrm{SP}}(q) \\
S_{\mathrm{CP}-\mathrm{CP}}(q) \\
S_{\mathrm{SP}-\mathrm{CP}}(q)
\end{array}\right],
\end{aligned}
$$

where the $4 \times 3$ matrix in the first line is denoted by $\mathbf{M}$. The matrix elements are composed of contrast factors, as follows:

$$
\begin{gathered}
\Delta_{\mathrm{SP}-\mathrm{SP}}\left(P_{\mathrm{H}, i}\right)=\left[\rho_{\mathrm{SP}}-\rho_{\mathrm{SBR}}\left(P_{\mathrm{H}, i}\right)\right]^{2} \\
\Delta_{\mathrm{CP}-\mathrm{CP}}\left(P_{\mathrm{H}, i}\right)=\left[\rho_{\mathrm{CP}}-\rho_{\mathrm{SBR}}\left(P_{\mathrm{H}, i}\right)\right]^{2} \\
\Delta_{\mathrm{SP}-\mathrm{CP}}\left(P_{\mathrm{H}, i}\right)=2\left[\rho_{\mathrm{SP}}-\rho_{\mathrm{SBR}}\left(P_{\mathrm{H}, i}\right)\right]\left[\rho_{\mathrm{CP}}-\rho_{\mathrm{SBR}}\left(P_{\mathrm{H}, i}\right)\right]
\end{gathered}
$$

Depending on our experiments, $\mathbf{M}$ forms a non-square matrix (the number of experiments corresponds to that of the rows). Therefore, instead of a simple inverse matrix, we need to employ the method of the Moore-Penrose pseudo-inverse matrix, $\mathbf{M}^{+}$, defined as follows:

$$
\mathbf{M}^{+}=\left(\mathbf{M}^{\mathrm{T}} \mathbf{M}\right)^{-1} \mathbf{M}^{\mathrm{T}}
$$

where $\mathbf{M}^{\mathrm{T}}$ is the transposed matrix of $\mathbf{M} . \mathbf{M}^{+}$is known to give the shortest-length least-squares solution for equation (18), as follows:

$$
\mathbf{M}^{+}\left[\begin{array}{c}
I\left(q ; P_{\mathrm{H}, 1}\right) \\
I\left(q ; P_{\mathrm{H}, 2}\right) \\
I\left(q ; P_{\mathrm{H}, 3}\right) \\
I\left(q ; P_{\mathrm{H}, 4}\right)
\end{array}\right]=\left[\begin{array}{c}
S_{\mathrm{SP}-\mathrm{SP}}(q) \\
S_{\mathrm{CP}-\mathrm{CP}}(q) \\
S_{\mathrm{SP}-\mathrm{CP}}(q)
\end{array}\right] .
$$

We evaluated $\mathbf{M}^{+}$for the four different $P_{\mathrm{H}}(0,29,38$ and $-34 \%$ ) and the obtained plots of $S_{\mathrm{SP}-\mathrm{SP}}, S_{\mathrm{CP}-\mathrm{CP}}$ and $S_{\mathrm{SP}-\mathrm{CP}}$ are shown by the symbols in Fig. 14(b). The partial scattering function of silica, $S_{\mathrm{SP}-\mathrm{SP}}$, agreed well with the spherical form factor, the same as that for SBR/SP. The partial scattering function of $\mathrm{CB}, S_{\mathrm{CP}-\mathrm{CP}}$, indicated a power-law function of $q^{-3.6}$, deviating from the Porod law $\left(q^{-4}\right)$ (Porod, 1951). This originates from surface structure of $\mathrm{CB}$. The result agrees with reports for CB-filled rubber specimens (Koga et al., 2005, 2008). The decomposition into partial scattering functions was successfully achieved. The cross-correlation term between silica and $\mathrm{CB}, S_{\mathrm{SP}-\mathrm{CP}}$, was also determined. $S_{\mathrm{SP}-\mathrm{CP}}$ is negligibly small compared with $S_{\mathrm{SP}-\mathrm{SP}}$ and $S_{\mathrm{CP}-\mathrm{CP}}$.

\section{Concluding remarks}

In this paper, we have reported the first attempt to use DNP and contrast variation SANS experiments on model mixtures for industrial tyres conducted at the MLF of J-PARC. We performed TOF-SANS experiments, employing neutrons with a wide $\lambda$ range, which causes imperfect neutron polarization and variations in the coherent and incoherent scattering lengths. By carefully eliminating the effect of imperfect neutron polarization, separation of the partial scattering functions was successfully demonstrated for the ternary system $\mathrm{SBR} / \mathrm{SP} / \mathrm{CP}$.

\section{Acknowledgements}

The neutron scattering experiment at the MLF of J-PARC was performed under the user programme (proposal No. 2016A0160). We appreciate the help of the MLF instrument safety team and sample environment team. This study was financially supported by a Grant-in-Aid for Young Scientists (A) (grant No. 25706033) of the Japan Society for the Promotion of Science.

\section{References}

Abragam, A. \& Goldman, M. (1978). Rep. Prog. Phys. 41, 395-467. Bouty, A., Petitjean, L., Degrandcourt, C., Gummel, J., Kwaśniewski, P., Meneau, F., Boué, F., Couty, M. \& Jestin, J. (2014). Macromolecules, 47, 5365-5378.

Brandt, B. van den, Glättli, H., Grillo, I., Hautle, P., Jouve, H., Kohlbrecher, J., Konter, J. A., Leymarie, E., Mango, S., May, R. P., Michels, A., Stuhrmann, H. B. \& Zimmer, O. (2006). Eur. Phys. J. $B, 49,157-165$.

Brandt, B. van den, Glättli, H., Hautle, P., Kohlbrecher, J., Konter, J. A., Michels, A., Stuhrmann, H. B. \& Zimmer, O. (2007). J. Appl. Cryst. 40, s106-s110.

Bunyatova, E. I. (2004). Nucl. Instrum. Methods Phys. Res. Sect. A, 526, 22-27.

Busfield, J. J. C., Deeprasertkul, C. \& Thomas, A. G. (2000). Polymer, 41, 9219-9225.

Byers, J. T. (2002). Rubber Chem. Technol. 75, 527-548.

Fermon, C., Glättli, H., van der Grinten, M., Eisenkremer, M. \& Pinot, M. (1992). Phys. B Condens. Matter, 180-181, 991-992.

Genix, A. \& Oberdisse, J. (2015). Curr. Opin. Colloid Interface Sci. 20 , 293-303.

Grinten, M. G. D. van der, Glättli, H., Fermon, C., Eisenkremer, M. \& Pinot, M. (1995). Nucl. Instrum. Methods Phys. Res. Sect. A, 356, 422-431.

Knop, W., Hirai, M., Schink, H.-J., Stuhrmann, H. B., Wagner, R., Zhao, J., Schärpf, O., Crichton, R. R., Krumpolc, M., Nierhaus, K. H., Rijllart, A. \& Niinikoski, T. O. (1992). J. Appl. Cryst. 25, 155165.

Koga, T., Hashimoto, T., Takenaka, M., Aizawa, K., Amino, N., Nakamura, M., Yamaguchi, D. \& Koizumi, S. (2008). Macromolecules, 41, 453-464.

Koga, T., Takenaka, M., Aizawa, K., Nakamura, M. \& Hashimoto, T. (2005). Langmuir, 21, 11409-11413.

Kohgi, M., Ishida, M., Ishikawa, Y., Ishimoto, S., Kanno, Y., Masaike, A., Masuda, Y. \& Morimoto, K. (1987). J. Phys. Soc. Jpn, 56, 26812688.

Koizumi, S., Iwase, H., Suzuki, J., Oku, T., Motokawa, R., Sasao, H., Tanaka, H., Yamaguchi, D., Shimizu, H. M. \& Hashimoto, T. (2007). J. Appl. Cryst. 40, s474-s479.

Kumada, T., Noda, Y., Hashimoto, T. \& Koizumi, S. (2009a). Nucl. Instrum. Methods Phys. Sect. Res. A, 606, 669-674. 
Kumada, T., Noda, Y., Hashimoto, T. \& Koizumi, S. (2009b). Phys. B Condens. Matter, 404, 2637-2639.

Kumada, T., Noda, Y., Koizumi, S. \& Hashimoto, T. (2010). J. Chem. Phys. 133, 054504.

Noda, Y., Kumada, T., Hashimoto, T. \& Koizumi, S. (2009). Phys. B Condens. Matter, 404, 2572-2574.

Noda, Y., Kumada, T., Hashimoto, T. \& Koizumi, S. (2011). J. Appl. Cryst. 44, 503-513.

Noda, Y., Yamaguchi, D., Hashimoto, T., Shamoto, S., Koizumi, S., Yuasa, T., Tominaga, T. \& Sone, T. (2013). Phys. Procedia, 42, 52-57.

Porod, G. (1951). Kolloid Z. 124, 83-114.

Schaefer, D. W., Rieker, T., Agamalian, M., Lin, J. S., Fischer, D., Sukumaran, S., Chen, C., Beaucage, G., Herd, C. \& Ivie, J. (2000). J. Appl. Cryst. 33, 587-591.

Sears, V. F. (1992). Neutron News, 3(3), 26-37.

Shibayama, M., Nagao, M., Okabe, S. \& Karino, T. (2005). J. Phys. Soc. Jpn, 74, 2728-2736.
Shinohara, T., Suzuki, J., Oku, T., Takata, S., Kira, H., Suzuya, K., Aizawa, K., Arai, M., Otomo, T. \& Sugiyama, M. (2009). Phys. B Condens. Matter, 404, 2640-2642.

Shinohara, T., Takata, S., Suzuki, J., Oku, T., Suzuya, K., Aizawa, K., Arai, M., Otomo, T. \& Sugiyama, M. (2009). Nucl. Instrum. Methods Phys. Res. Sect. A, 600, 111-113.

Stapf, S. \& Kariyo, S. (2005). Acta Phys. Pol. A, 108, 247-259.

Stuhrmann, H. B. (2015). J. Optoelectron. Adv. Mater. 17, 14171424.

Stuhrmann, H. B., Schärpf, O., Krumpolc, M., Niinikoski, T. O., Rieubland, M. \& Rijllart, A. (1986). Eur. Biophys. J. 14, 1-6.

Takata, S., Suzuki, J., Shinohara, T., Oku, T., Tominaga, T., Ohishi, K., Iwase, H., Nakatani, T., Inamura, Y., Ito, T., Suzuya, K., Aizawa, K., Arai, M., Otomo, T. \& Sugiyama, M. (2015). JPS Conf. Proc. 8, 036020.

Takenaka, M., Nishitsuji, S., Amino, N., Ishikawa, Y., Yamaguchi, D. \& Koizumi, S. (2009). Macromolecules, 42, 308-311. 